This is a pre-print of an article published in Software Quality Journal. The final authenticated version is available online at: https://doi.org/10.1007/s11219-020-09505-2

\title{
RETORCH: An Approach for Resource-aware Orchestration of End-to-End Test Cases
}

\author{
Cristian Augusto [0000-0001-6140-1375] $1($ 凹) ,Jesús Morán [0000-0002-7544-3901] 1, Antonia Berto- \\ lino [0000-0001-8749-1356] 2 , Claudio de la Riva [0000-0001-5592-9683] 1, Javier Tuya [0000-0002-1091- \\ 934X] 1 \\ ${ }^{1}$ Computer Science Department, University of Oviedo, Gijón, \\ ${ }^{2}$ ISTI-CNR, Consiglio Nazionale delle Ricerche, Pisa, Italy \\ ${ }^{1}$ \{augustocristian, moranjesus, claudio, tuya\}@uniovi.es \\ 2 antonia.bertolinodisti.cnr.it
}

\begin{abstract}
Continuous integration practice mandates to continuously introduce incremental changes into code, but doing so may introduce new faults too. These faults could be detected automatically through regression testing, but this practice becomes prohibitive as the cost of executing the tests grows. This problem is preponderant in End-to-End testing where the whole system is requested for test execution. However, some of these test cases could be executed with fewer resources (e.g. memory, web services, computation, Cloud instances, among others), by deploying only the subsystems needed by each test. This paper is focused on the optimization of the resources employed in End-to-End testing by means of a resource-aware test orchestration technique in the context of continuous integration practices in the Cloud. The RETORCH approach proposes a novel way to identify the resources required by End-to-End test cases and to use this information to group together those tests requiring equivalent resources. Besides, the approach proposes to deploy the grouped tests in isolated and elastic environments, so that their execution can be scheduled in parallel on several machines. RETORCH is exemplified with a real-world application and its performance evaluation shows promising savings in terms of resource usage and time.
\end{abstract}

Keywords: Software testing, Continuous integration, Continuous testing, Testing in the cloud, End-to-End testing, Test orchestration.

\section{Acknowledgements}

This work was supported in part by the Spanish Ministry of Economy and Competitiveness under TestEAMoS (TIN2016-76956-C3-1-R) project and ERDF funds, and by the European Project ElasTest in the Horizon 2020 research and innovation program (GA No. 731535).

This is a pre-print of an article published in Software Quality Journal. The final authenticated version is available online at: https://doi.org/10.1007/s11219-020-09505-2 


\section{Introduction}

Continuous integration practices are based on incremental changes in the code to improve quality or add new functionalities (Meyer, 2014). However, while introducing new features in the code, new faults can be introduced as well. Detect these failures early in the code may reduce between $25-40 \%$ the amount of time and cost in fixing them compared to fix them in production (Shull et al., 2002). To ensure that the modifications and the new code do not endanger the existing functionality, regression testing (Yoo \& Harman, 2012) is standard practice. In modern agile processes, though, in which new versions of software are continuously and frequently delivered within very short cycles, regression testing may face many challenges as the efficient execution of the test cases, the reliability improvement of the tested system or the reduction of time between different releases.

One emerging practice to shorten the validation of newly released versions is continuous testing (Fitzgerald \& Stol, 2017). Continuous testing consists of automating the test cases and re-executing them before any new release in the source code repository. However, a well-known problem is that as the number of tests increases, re-executing all of them at each change may not be possible due to the extent of resources that should be employed, such as the computational execution cost, the time required, or the number of instances needed. As a solution to partially address this problem, many test minimization and prioritization techniques (Yoo \& Harman, 2012) have been proposed, to identify a minimal subset of test cases or optimize their order of execution, respectively. The objective of these techniques is to look for a tradeoff between the probability of discovering the faults potentially added with modifications and the resources employed for regression testing. The prioritization techniques permute the execution order of the test cases aimed to firstly execute the most relevant test cases, but the whole execution of the test suite remains expensive unless the tester decides to execute only a subset of the more relevant test cases through a minimization technique. The latter techniques reduce the execution time by not re-executing all test cases, but they neither optimize the resources of the test executed nor alleviate the thoroughly use of resources in the whole test suite.

One of the testing levels that require a large amount of physical-logical-computational resources is End-to-End testing (from now onwards referred to as E2E). E2E testing includes the interaction between system components, from the user interaction with the system to low-level layers like databases. The execution of the E2E test cases requires large amounts of resources due to the high execution time, the cost of replicating resources, or the set-up of the system, among others. Therefore, the application of techniques such as prioritization minimization or reduction may not be effective enough for cost reduction in E2E testing.

During the execution of E2E test cases, the resources are usually oversubscribed because the test cases tend to deploy more resources than they need for execution. For example, suppose a User Interface test case that requires a web and database server, but the set-up of this test case deploys the whole system including an email server. As a consequence, the test case oversubscribes resources because the deployment of the whole system also includes an email server that this test will not use.

This is a pre-print of an article published in Software Quality Journal. The final authenticated version is available online at: https://doi.org/10.1007/s11219-020-09505-2 
A proper set-up of the whole system is relevant not only to optimize the resources deployed during E2E testing but also to decrease the execution time. Thus, if this setup requires a large amount of time compared with that employed in test execution, parallelizing the test cases in separate instances without a proper strategy would not solve the problem: for the test cases that share the usage of heavy resources, parallelization would be inefficient and the best solution would be to set-up the test environment once and execute them in a sequential way. Therefore, to optimize the cost of E2E testing, the detection of the dependencies between the test cases and the resources is a crucial aspect which may achieve cost savings (Herzig, Greiler, Czerwonka, \& Murphy, 2015).

Moving testing to the Cloud (Bertolino et al., 2019) is commonly acknowledged as a solution to reduce the cost of testing, especially to exploit the potential of unlimited resources and scalability delivered on demand. One open-source platform to support Cloud testing and simplify the E2E testing process has been developed within the European Project ElasTest (Bertolino et al., 2018) The solution avoids several testing dependence problems by providing dependency isolation through the containerized execution of the tests. This is done through the TJobs that are the tests together with the Docker containerized system under test (SUT) customized to provide not only the production environment but also utilities to execute, monitor and collect testing information.

Containerization has provided new advantages in the virtualization field, reducing the amount of both resources and time required to deploy a service in an isolated environment. The SUT instantiation can take advantage of the containerization in order to be deployed several times in the same machine, avoiding common problems like dependencies. However, in the current version, the ElasTest containerized execution presents the problem that it needs the instantiation of the resources required for each container causing oversubscribing (under usage) of those resources.

Our proposal is intended to reduce the number of resources used in the containerized execution of the test during E2E testing and it may be integrated into the ElasTest platform to support resource-aware Cloud testing orchestration: we call the approach RETORCH (Resource-aware E2E Test ORCHestration). RETORCH aims at decreasing the execution time and optimizing the resources used in E2E testing through the identification of the resources used during testing, groups the tests based on the resources they need to avoid unnecessary re-deployments and running of the identified test groups in parallel to reduce the execution time.

This article extends an earlier work (Augusto, Morán, Bertolino, de la Riva, \& Tuya, 2019) by introducing a number of new concepts that are useful to identify the resources used by the test cases, a complete reorganization and extension of the state-of-the-art, and the application and evaluation of the approach on an Elastest demonstrator. More precisely, this article includes the following contributions:

1. Definition of the RETORCH framework to perform the E2E resource identification, the test grouping and scheduling.

2. An illustrative application scenario of RETORCH usage.

3. An evaluation of the RETORCH approach in a real-world application.

This is a pre-print of an article published in Software Quality Journal. The final authenticated version is available online at: https://doi.org/10.1007/s11219-020-09505-2 
The remainder of the article is organized as follows. The related work is described in Section 2. The orchestration approach proposed in this article is defined in Section 3. Section 4 describes a working example related to a teaching online service (FullTeaching application using the OpenVidu Streaming Engine). Section 5 contains the evaluation of the approach proposed using a real-world application. Finally, the conclusions and future work are in Section 6.

\section{Related work}

An inspiring work to RETORCH is the Multi-Objective Regression Test Optimization approach (Harman, 2011). In his work, Harman discussed several cost and value-based objectives for testing, supporting the point of view that testing optimization should be performed by considering a combination of the several different types of resources needed. Our work is also focused on the same problem and proposes a specific solution for the case of End to End testing, considering a number of resources and the time spent during the testing. This section discusses different lines of orthogonal works that are related to RETORCH: (1) the reduction, prioritization and minimization techniques, (2) test dependency detection, (3) the resource optimization techniques and finally (4) the orchestration approaches.

\subsection{Test Reduction, Prioritization and Minimization Techniques}

Despite the recent advances in both efficiency and effective usage of resources during the testing, there are several open challenges (Bertolino, 2007) to be addressed when performing test prioritization, selection, and minimization. Test reduction, prioritization and minimization have been widely discussed in the literature. Yoo and Harman (Yoo and Harman 2012) have surveyed some works about minimization, prioritization and selection, comparing the results of these techniques in terms of failure detection effectiveness and discussing open problems and future directions of them. Several authors have studied approaches to optimize these techniques considering both cost and rate of fault detection (Engström, Skoglund, \& Runeson, 2008; Rothermel, Harrold, Von Ronne, \& Hong, 2002; Wong, Morgan, London, \& Mathur, 1998). These techniques are also used in big companies like Google (Memon et al., 2017) that executes a subset of the test cases according to both the failure rate and the historical number of modifications. Another line of research combines these approaches with other techniques, such as Machine Learning (Lachmann, Nieke, Seidl, Schaefer, \& Schulze, 2017), prioritizing the test cases according to the requirements coverage, execution cost and the historical number of failures detected by the test cases. (Yoo \& Harman, 2012)

Our proposal has some aspects in common with the arrangement of the test cases of prioritization techniques (Yoo \& Harman, 2012).

This is a pre-print of an article published in Software Quality Journal. The final authenticated version is available online at: https://doi.org/10.1007/s11219-020-09505-2 


\subsection{Test Dependency detection}

During the test prioritization, one relevant issue to consider is the test dependencies. Some authors have proposed techniques and tools to detect these dependencies between test cases. Bell et al. (Bell, Kaiser, Melski, \& Dattatreya, 2015) provide a tool to detect dependencies (Electrictest), and compare it with other state-of-the-art tool achieving similar fault detection rate but with lower slowdown. The Electrictest tool was evolved by Gambi et al. (Gambi, Bell, \& Zeller, 2018) and tested empirically achieving good results: they discover several dependencies previously known and also another one never discovered by the previous tests and tools. Gyori et al. (Gyori, Shi, Hariri, \& Marinov, 2015) have introduced the concept of the test pollution problem and present a technique (called POLDET) which was implemented into a tool that detects in execution time the "polluting" tests.

Our article proposes a framework to optimize the resources of the test executions avoiding unnecessary system re-deployments by grouping those test cases that have no dependencies between them. The test dependencies play a key role to discover the relationships between the test cases and their resources. Our approach is intended to introduce a dependence detection mechanism to improve the test efficiency though the aggrupation of those test cases that they do not interfere with their execution.

\subsection{Test Resource Optimization}

The optimization of the test resources has been widely covered in the literature. Several works attempt to choose between different objectives as optimize the time, cost or a mix of both (Gambi, Gorla, \& Zeller, 2017), or optimize the resources in testing at the same time that they comply with time constraints (Liu, Chen, \& Chen, 2017). Other authors (Chakraborty \& Shah, 2011) have focused on the cost optimization, via different processes that partition, group and redistribute the test cases in order to parallelize them. This aggrupation or partition of test cases are also present in clustering techniques applied to the test optimization problem (Yu, Su, \& Wang, 2009), on which the resources are linked with the test cases in order to discover the underlying dependencies and execute them.

Unlike the previous works, our article proposes to optimize the resources not only focused on time or cost but also on other resources used during the testing based on both the test dependencies and resource usage. García et al. (Garcia et al., 2018) also propose to orchestrate the test cases through a proper selection and sequencing based on the outcome of test execution (verdict-driven) or on the produced output (datadriven).

In the field of Cloud services, several approaches have been proposed to face similar issues related to resource optimization. The Microsoft CloudBuild (Esfahani et al., 2016) faces dependences issues extracting dependency graphs and deriving the dependencies on them automatically. Based on these dependency analyses, Microsoft CloudBuild optimizes the testing execution through the deployment and execution of only the test cases that change the code. We propose a future line of work that aims at a similar automated detection of the test resources into the containers.

This is a pre-print of an article published in Software Quality Journal. The final authenticated version is available online at: https://doi.org/10.1007/s11219-020-09505-2 


\subsection{Orchestration}

Depending on the field, the term orchestration has different meaning and usage. In general, orchestration is a jargon term that refers to the action of coordinating and scheduling several components improving their execution. In Network field, Giotis et al. (Giotis, Kryftis, \& Maglaris, 2015) propose to orchestrate the Network Functions Virtualization (NFV) with the goal of managing a policy-based traffic engineering. In the cloud field there are a number of orchestration systems, as for example Kubernetes (Burns, Grant, Oppenheimer, Brewer, \& Wilkes, 2016), Borg (Burns et al., 2016), Swarm (Docker Inc., 2019), Fuxi (Zhang et al., 2014), System Center-Orchestrator (Microsoft, n.d.). These previous orchestration systems are focused on Cloud following different architecture like TOSCA (Cloudify and Kubernetes) (Draft, 2014).

The orchestration in Cloud is performed via an orchestrator (e.g. Docker Compose (Docker Inc., 2017)) that monitors and deploys the Jobs focused on optimizing the usage of the instances (Casalicchio, 2017) or providing a determined QoS (Singh \& Chana, 2015).

Closely related to Cloud services, Fog and Edge Computing also address several new open challenges related to resource optimization (Velasquez et al., 2018). These challenges are usually addressed via different architectures that present an orchestrator acting as both allocator and scheduler of the resources available (De Brito et al., 2017; Velasquez et al., 2017). Several of these works were analyzed according to the resource scheduling, allocation, sharing, or optimization by Tozé et al. (Toczé \& Nadjm-Tehrani, 2018) proposing a Taxonomy of resource management in the Edge.

These works propose orchestration techniques to optimize the resources according to their specific domain. Instead, our approach aims to optimize the resources employed in the execution of the E2E testing thus decreasing the execution time while achieving savings in the usage of resources.

\section{RETORCH Overview}

The RETORCH framework aims at optimizing the cost/usage of resources orchestrating the E2E test cases in different machines based on the resources needed to execute each test. Fig.1 depicts the core concept of the orchestration starting from the E2E test cases to their execution in several machines/instances grouping those tests that use homogeneous resources in order to optimize both resources and execution time.

This is a pre-print of an article published in Software Quality Journal. The final authenticated version is available online at: https://doi.org/10.1007/s11219-020-09505-2 


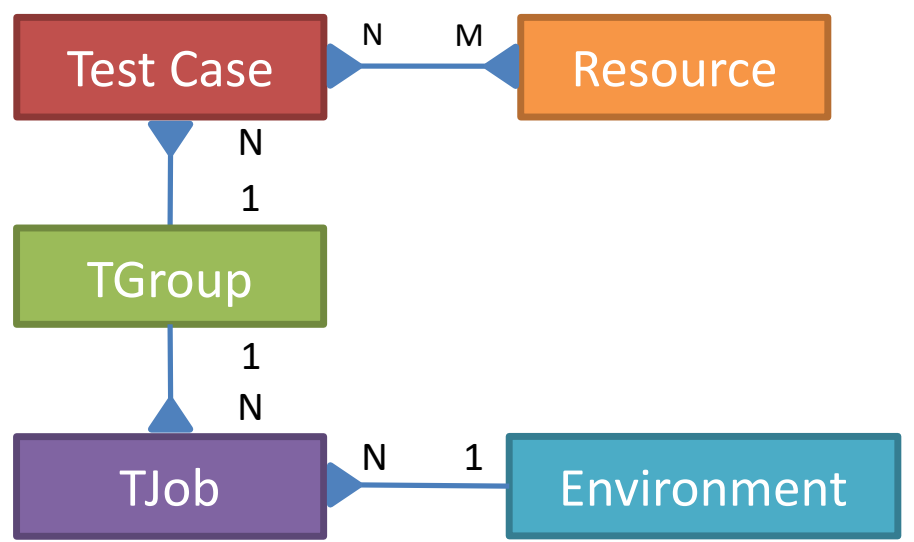

Fig. 1. Key concepts of RETORCH

As the first step, resources used by each test case are identified to detect which test cases require the same resources (Resource identification). According to the resources identified, some tests can be executed together while others cannot because of incompatibilities in their allocated resources or in the way in which these tests access the resources. Then, those test cases that can be executed together are grouped to arrange their execution and reuse their resources to optimize their cost (Grouping). These groups of tests are called TGroups. Test cases that belong to different TGroups can be executed independently because the resources they employ are different. Finally, each TGroup may be split and allocated in several instances (Scheduling) to optimize both the cost/usage of resources and the test execution time. The test cases of these TGroups are split into several disjoint subsets, which are smaller than the previous TGroups, which are called TJobs. Each TJob contains not only the code of a subset of test cases but also the environment with the dependencies isolated in a container that allows easy deployment of the test cases in a Cloud instance.

In the following subsections, the above key concepts are detailed. Subsection 3.1 details the resources and their attributes. These resources can be characterized according to their category (Subsection 3.1.1), the static attributes (Subsection 3.1.2), and the dynamic attributes that change during test execution (Subsection 3.1.3). Finally, the processes that orchestrate the E2E test cases are defined in Subsection 3.2.

\subsection{Main concepts of RETORCH}

The core of RETORCH is based on four main concepts defined below: the resources required by the test cases, the groups of these test cases that can re-use the same resources (TGroups), the disjoint subsets of these TGroups in which each subset can be allocated independently in parallel to decrease the execution time (TJobs), and an

This is a pre-print of an article published in Software Quality Journal. The final authenticated version is available online at: https://doi.org/10.1007/s11219-020-09505-2 
environment to isolate the dependencies to allow the scheduling in elastic Cloud instances.

- Resources are physical, logical and/or computational entities required by the execution of one or more test cases. Examples of resources are databases with their tables, web Servers, mobile phones, services such as a payment gateway or a pool of containers provided by a Cloud carrier.

- TGroup (Test Group): is a set of test cases that use homogeneous resources and can be deployed together in the same environment. For example, a TGroup can contain the test cases that query the same database with the same initial load and without modifying the information. These test cases can use the same database setup in the same instance. In contrast, if two test cases modify the database causing side effects like flakiness or other issues related to dependencies, then these two test cases must be on different TGroups and therefore deployed in different environments. Each TGroup settles the environments needed by the test execution in the whole system or also considering scaffolding and test harness through the mocks, stubs or other simulators that can alleviate the cost of resources that are not mainly needed for the tests of the TGroup. The test cases of the TGroup can be also divided to not only optimize the cost/usage of resources but also the execution time through a distributed scheduling.

- TJob (Test Job): is a subset of a TGroup containing several test cases inside a Docker container that also deploys the environment composed by the system under test isolating the dependencies and customized to provide utilities to execute, monitor and collect testing information. The TGroup are split into several TJobs which are scheduled into different Cloud instances to reduce the execution time due to the parallel execution. Each TJob contains a subset of test cases that also re-use the same resources to optimize the resource usage avoiding unneeded re-deployments of the environment.

- Environment is the set of resources requested and which they interact with a test case during testing. Examples of environments are a web application composed by a web server and a database or several mobile phones required to test an Android application.

The following subsections define the categories of the resources (Subsection 3.1.1), their static attributes (Section 3.1.2) and the attributes relative to the usage of the resources during E2E testing (Subsection 3.1.3).

\subsubsection{Resource Categories}

Resources are classified according to three different categories described below:

1) Physical: the tangible resources that are used during E2E testing. For example, the smartphones used to test a mobile application, the router that allows us to configure a virtual network into a system test, or other peripherals like printers, sensors, among others.

This is a pre-print of an article published in Software Quality Journal. The final authenticated version is available online at: https://doi.org/10.1007/s11219-020-09505-2 
2) Logical: the non-tangible resources used during E2E testing that are provided in the traditional way. For example, a web server on which the application under test is deployed, the operative system on which the test is performed or one flight emulator that simulates a plane in ATM.

3) Computational: the logical resources that are provided or consumed as a service. This category consists of those resources served on-demand in Cloud models like IAAS (Infrastructure as a Service), PAAS (Platform as a service) or SAAS (Software as a service), on which the computation, software, network or storage, may be provided as needed by the tester.

\subsubsection{Static Attributes}

Regardless of the category, the resources are also characterized according to certain static attributes. These static attributes do not change during the E2E execution and provide additional information about the resource and how it can be used during the testing. We consider the following static attributes:

- Elasticity: A resource is elastic when it can be instantiated and made available for the tests cases on the fly (e.g. a database running in a container, a software simulator). Conversely, a resource is not elastic when only a fixed maximum number of instances are available (e.g. a sensor, a camera, a hardware emulator).

- Hierarchy/partitioning: A resource may contain sub-resources or partitions that are also resources (e.g. a database may be partitioned into several tables or sets, of tables). These sub-resources and partitions characterize the resources.

- Sharing: Shared resources may be used simultaneously by more than one test case without interfering into the test result.

- Replaceable: One resource is replaceable if may be interchangeable by a new instance (or another equivalent resource) with no penalty for a given test case. For example, one service that only provides tokens is replaceable if we can replace it by a simple mock that also provides continuously the same token for all requests in a similar way than the original resource.

- Lifecycle: All resources have a lifecycle composed of different phases like the setup of resource, test execution using the resource and disposal of the resource. In the set-up phase, the resources are deployed and initialized according to the test data (e.g. initial load of the database or configuration data). Once the resources are ready, the test cases use them during the test execution. Finally, after the test execution has finished, the resources are disposed and released, making them available for other test cases into a disposal phase.

For example, suppose the E2E testing of an Air Traffic Management (ATM). When testing the operations that an air traffic controller makes to manage their assigned flights, we need a resource that is the Control Working Position (CWP), which is itself a complex non-elastic physical system. The CWP may become a shared resource if we partition the flight area (a logical resource) into hierarchical clusters of sectors, provided that each test case will manage only flights belonging to a cluster. Moreover, when testing a transfer of flights between controllers will need two CWP, either

This is a pre-print of an article published in Software Quality Journal. The final authenticated version is available online at: https://doi.org/10.1007/s11219-020-09505-2 
exclusive or shared. The CWP is a not replaceable resource because is a highly coupledcomplex system that makes even more complicated deploy partially its functionalities or change it by a mock. This resource also has his own lifecycle, with a set-up (prepare all the CWP and flight plans), a test phase and finally a release and disposal.

\subsubsection{Access Mode and Dynamic Attributes}

The resources can also be characterized according to their usage during the E2E testing considering how each test case accesses the resource (access mode) and how the resource changes due to the test execution.

Each test case uses the resources through different operations characterized by two properties: safety and idempotency. Safe operations are those whose execution does not modify the resource, for example, a SELECT or a JOIN operation in a database query because it does not change the information of the database and does not introduce dependencies between test cases. Idempotent operations are those that can be performed several times consecutively producing the same result.

Different test cases may have different usage patterns when using the same resource. Each pair of test case and resource is associated according to an access mode that determines if the operations performed during the test execution modify the resource or not, and how. The access modes are enumerated below:

- Read-only: the test case performs both safe and idempotent operations allowing other test cases to read the resource at the same time (e.g. a test case that queries the master tables of a database without any change, allows that other test cases query the same resource).

- Read-write: the test case performs operations that are neither safe nor idempotent. Then, other test cases may not use this resource simultaneously to avoid unexpected erroneous executions (e.g. all half-duplex communication channel, on which two devices can emit or receive, but not at the same time in the same channel)

- Write-only: the test case performs operations that are neither safe nor idempotent similar to those "read-write", but allows that more than one test case update the resource simultaneously, restricting reads to only assertions that check the expected results (e.g., a centralized log system that acts as a sink for several test cases, provided that, if we need to check the logs, there is a mechanism that allows identifying the logs produced by each test case).

- Dynamic: the test case performs operations that are safe but not idempotent. The resource is partitioned on the fly allowing that each test case create and access each partition independently from other test cases (e.g., when testing several test cases that issue orders, more than one test can place an order at the same time, but in dynamic access each test case must only use the orders that it has created).

- No access: This access mode is banally safe because the operations of the test case do not make use of the resource (e.g., when using a simple mock that does not require any resource).

This is a pre-print of an article published in Software Quality Journal. The final authenticated version is available online at: https://doi.org/10.1007/s11219-020-09505-2 
The previous characterization provides insights about how the E2E tests use the resources. During the test execution, other attributes may change dynamically due to the usage of the resource. These attributes are called dynamic and are the following:

- Allocated: Location of each resource must be known to make possible their identification (e.g., the environment over where is deployed). Allocation is crucial when an effective use and measure of the resource performance during testing is considered.

- Measurable: Each resource must have indicators to allow measuring how many of them are deployed and their performance (e.g. RAM, processor usage or heartbeat latency received by a sensor network).

- Elasticity Cost: The elasticity cost measures the expenses incurred during the resource lifecycle. This cost may be a combination of money, time, processing power, memory, energy, among others.

- Traceability: Each resource must be always traceable, allowing to know its state at every time of the test execution according to the lifecycle (e.g. ready, running, disposing of, or testing over it).

- Test Instance: The resources and test cases must be deployed in an instance that isolates the dependencies and avoids wrong executions/accesses with a properly setup.

- Availability: According to the number of instances available, resources are classified into renewable and non-renewable. Resources are renewable if can be re-instantiated without any kind of limitations. On the other hand, a resource is non-renewable when only may be instantiated a fixed amount of times.

- Granularity: Each resource has its own granularity depending on how the scope is focused over it and its underlying sub-resources. For example, one mobile phone that is used as a physical device for the testing may be considered with more granularity as a set of sub-resources (camera, microphone, screens, among others).

For example, in the previous scenario (ATM), the Flight plans in an Air Traffic simulator are usually shared and renewable resources, because they are created on the fly as needed when the test is performed. On the other hand, the operation logs that are kept for legal requirements, are a write-only resource because they do not use it for anything other than saving the different usage traceback.

\subsection{Processes}

RETORCH has three different processes, namely Resource Identification, Grouping and Scheduling. The resource identification provides insights about the resources required by the test cases and their dependencies. Next, the grouping is performed to group together those test cases that can be executed together to re-use resources. Finally, the scheduling optimizes the execution of the test cases providing a parallel schedule that reduces the test execution time. These processes are represented in Fig. 2 and described below:

This is a pre-print of an article published in Software Quality Journal. The final authenticated version is available online at: https://doi.org/10.1007/s11219-020-09505-2 


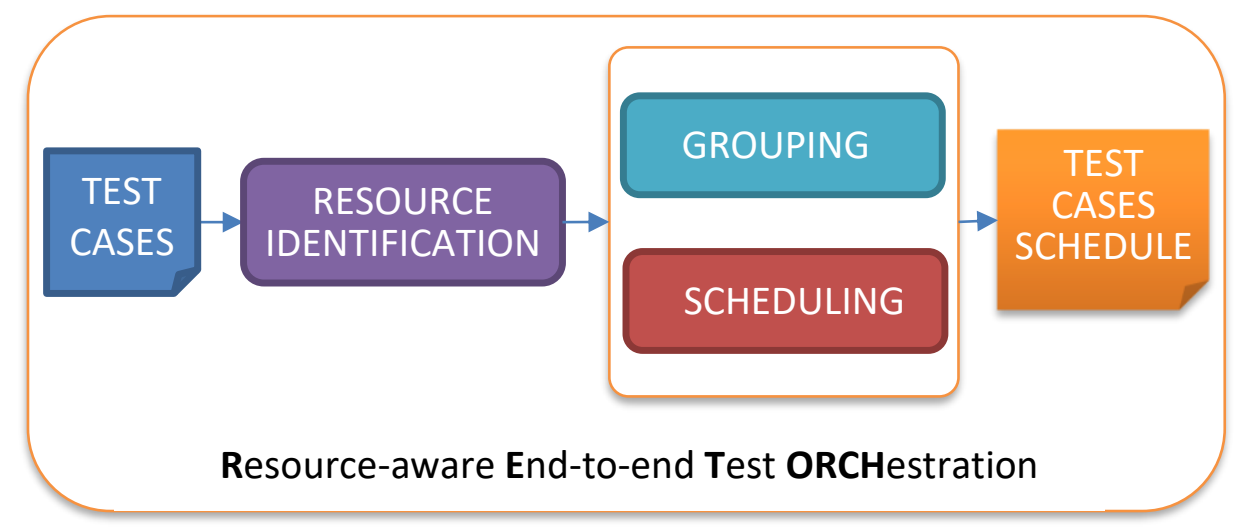

Fig. 2. Scheme of the main RETORCH processes

Resource Identification: This process identifies the resources that each test case needs to be executed properly. To determine how the test case uses the resource, each association of a resource and test case is labelled with an access mode and the attributes (Subsection 3.1). With all this information, the test cases are characterized obtaining all the resources and their attributes, which will be the basis for grouping and scheduling the test cases.

Grouping: This process aims at optimizing the usage of resources through an aggrupation of those test cases that can re-use the same resources to avoid unneeded redeployments. The test cases are arranged together into TGroups (Subsection 3.1) based on the compatibility of the attributes of the resources employed by these tests. The result of this grouping is a set of test cases together with all scaffolding required for the execution. The main goal of the grouping process is to avoid the oversubscription of the resources when one test case requests more resources than needed. For example, if two tests perform an operation with a safe access mode they can be grouped together. However, if two test cases perform a non-safe operation on the same resource, they are candidate to be placed in the same or separate groups depending on the access mode.

Scheduling: Although the grouping process achieves some optimization on resource usage, the whole test process may be further optimized by ordering and splitting the TGroups into a TJobs (Subsection 3.1). For instance, TJobs may be distributed in parallel to achieve better use of the test infrastructure and reduce the execution time. Not all schedules are aimed to minimize both execution time and the resource usage (one possible objective may be to maximize the usage of several instances, minimizing the idle time or another possible objective may be minimizing the execution time using more resources).

This is a pre-print of an article published in Software Quality Journal. The final authenticated version is available online at: https://doi.org/10.1007/s11219-020-09505-2 


\section{$4 \quad$ Working example}

To illustrate RETORCH, we present an example of its application on a real-world opensource application called FullTeaching (Pérez, 2017). FullTeaching is an educational platform that provides many features for organizing the teaching material, courses, and structuring classes; it provides also means for interacting with students, e.g., calendars, dashboards, forums.

Resource identification. The FullTeaching system is a resource that can be partitioned in hierarchical way by several sub-resources, including the OpenVidu videoconference server (University, 2017), the Kurento media server (Technologies, 2014), and the MySQL DBMS (Oracle, 2019). In particular, for online teaching, FullTeaching includes features enabling real-time video conferencing that are supported by OpenVidu via W3C Web-RTC (Uberti \& Thatcher, 2018) open-source API. For testing the E2E functionality, the testers should consider the underlying infrastructure and the usage of resources, especially for the most expensive one (OpenVidu).

Deploying one instance of the OpenVidu resource per each test case that requires this resource is too expensive due to heavy resources for storage and graphical processing evolved in the video streaming. Despite we can group test cases to re-use the OpenVidu deployments, the OpenVidu resource is replaceable because it can be changed by simple mock in some test cases. For example, the test cases that only use the OpenVidu resource to acknowledge the connection, they do not need the full Open$V i d u$ resource and can replace it by mock resource to be more efficient. Considering that the OpenVidu resource can be replaced based on how the test cases use this resource, we identify the following three replacements of the OpenVidu resource with different elasticity costs:

1. Light OpenVidu resource: This resource is a mock that just provides a random number as session-id, whenever any client requires it. Precisely, this resource has a No-Access mode meaning that the requests from the test do not access the real Open$V i d u$ resource, but a mock resource. This resource may be used by the test cases that only require the session-id from OpenVidu.

2. Medium OpenVidu resource: This resource is a simple implementation of the real OpenVidu resource, but with only basic functionalities and without any storage to record the session. This resource will be employed by the test cases that only need to check functionalities without storage like online chats between users or the navigation in the classroom menu.

3. Heavy OpenVidu resource: This resource provides all the functionalities of OpenVidu besides several video lessons recorded. This will be used in those test cases that require these video streaming recording functions or require all the functionality of the engine for their execution.

Once identified the previous three resources, we proceed to arrange all the test cases available depending on their resource usage requirements. Test cases assigned to a Light OpenVidu are the cheapest in term of elasticity cost: they can be available for testing on the fly and can be shared between multiple tests. The lifecycle of this resource This is a pre-print of an article published in Software Quality Journal. The final authenticated version is available online at: https://doi.org/10.1007/s11219-020-09505-2 


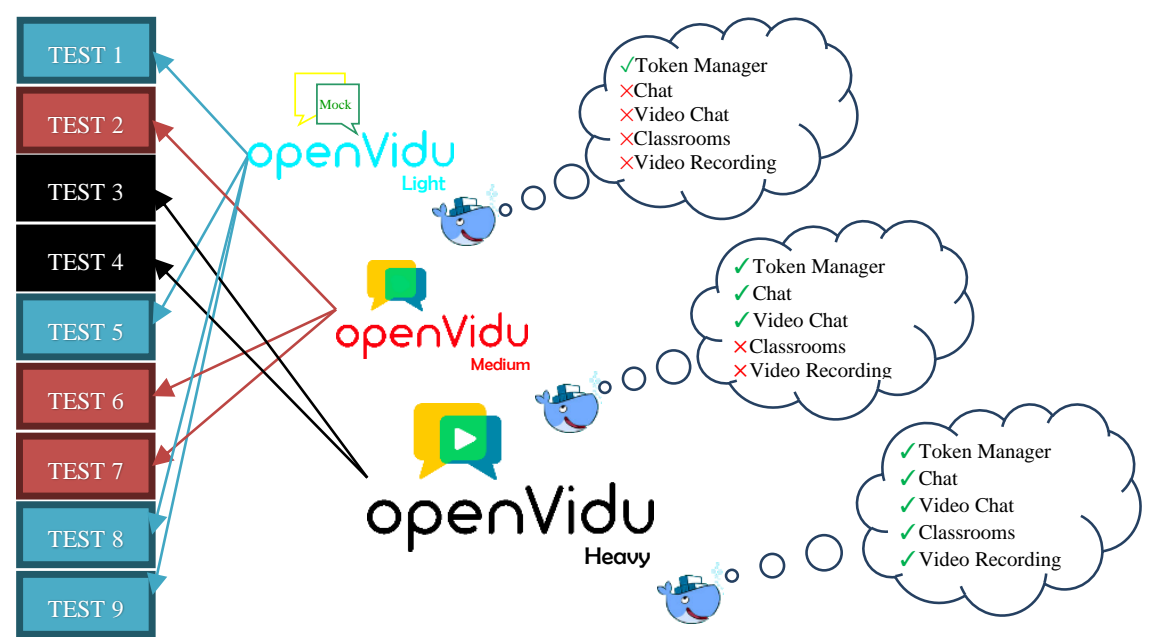

Fig. 3. Resource identification and grouping

differs that it does not require additional set-up or disposal, getting an improvement in terms of cost.

Test cases assigned to a Medium OpenVidu resource require the deployment of a simple container that consumes a small number of resources in terms of elasticity cost and it allows sharing between multiple tests (although with some performance penalty). In this case, the set-up/dispose lifecycle phases are more expensive than the light Open$V i d u$ resource, so the aggrupation of the test cases (Grouping) can reduce the usage of resources sharing this set-up between several tests. Test on this resource has a ReadWrite access mode.

Last, test cases assigned to the Heavy OpenVidu resource should be executed in a sequential way because they access to the resource in a read-write mode and the high elasticity cost that does not allow the deployment of more than one instance. This resource has this type of access mode because the test cases use the resource to create and modify videos at the same time. As a consequence, the test cases that use the Heavy OpenVidu resource should be executed sequentially to avoid issues due the concurrent access/modification of the same videos.

Grouping: Once the resources are identified and characterized, we proceed to group these test cases into TGroups considering the test dependencies with the resources used. Fig. 3. depicts the mapping between test cases and the groups (TGroup) that they belong to. The test cases that use the Light OpenVidu resource are represented in blue colour, the test cases that use the Medium OpenVidu resource in red colour, and the test cases that use the Heavy OpenVidu resource in black colour.

Let suppose that we have nine test cases and determine three TGroups as indicated below:

TGroup 1 (Light OpenVidu): Test case 1, 5, 8 and 9

TGroup 2 (Medium OpenVidu): Test case 2, 6 and 7

This is a pre-print of an article published in Software Quality Journal. The final authenticated version is available online at: https://doi.org/10.1007/s11219-020-09505-2 


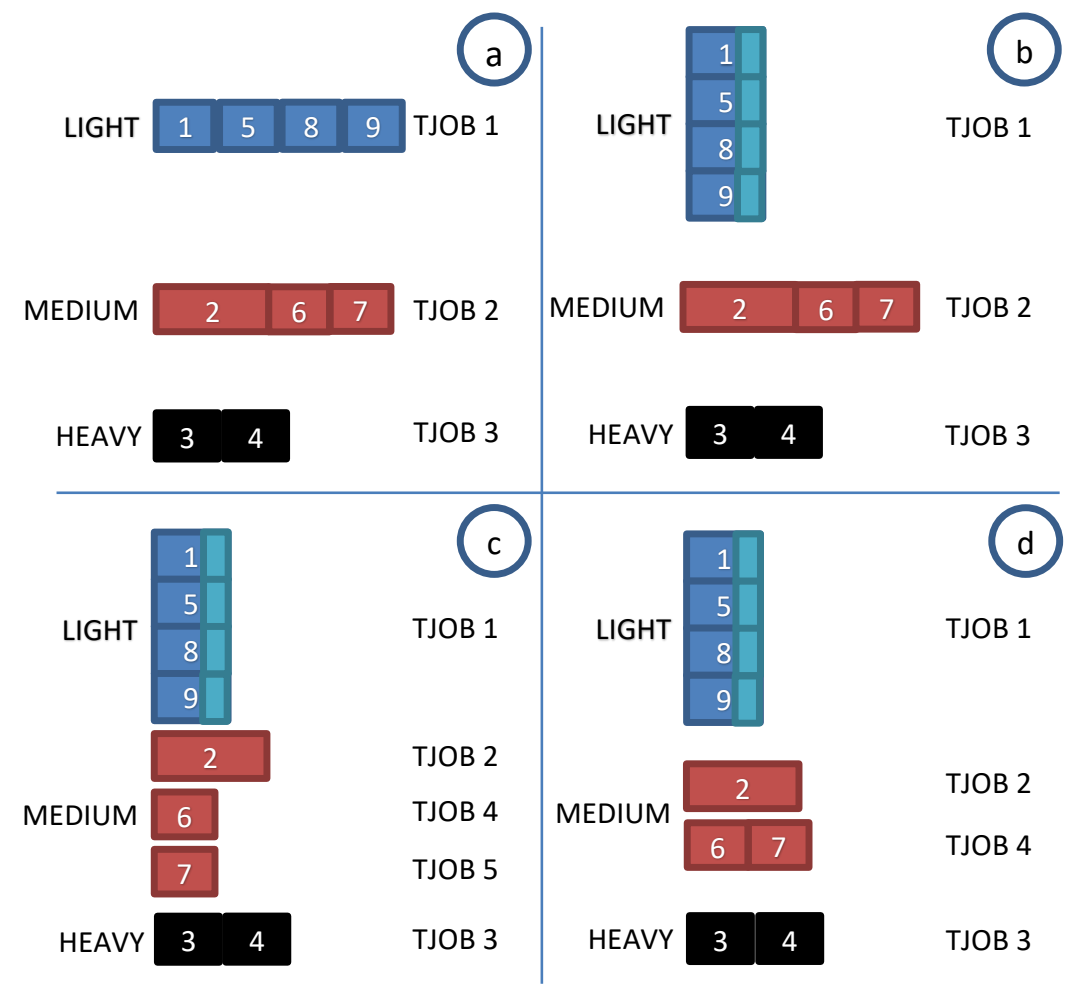

Fig. 4. Different proposed TJob Scheduling

\section{TGroup 3 (Heavy OpenVidu): Test case 3 and 4}

The previous aggrupations can improve resource usage deploying minimal resources and avoiding unnecessary re-deployments. For example, the TGroup 1 instead to deploy the OpenVidu resource, just deploy a mock resource (Light OpenVidu resource) that is more efficient in terms of resource usage. However, there are test cases that require the OpenVidu resource like Test case 3 and 4 and they deploy this whole resource (Heavy OpenVidu resource), but they can be executed in the same group (TGroup 3) to deploy the resource one time and re-use again avoiding a new unneeded re-deployment.

Scheduling: Once the grouping is done, we divide the TGroups into TJobs to schedule them and optimize both objectives: the resource usage and execution time. Fig.4 represents four different schedules. The TJobs derived from TGroup 1 are represented in blue (Light), the TJobs of TGroup 2 in red (Medium), and the TJobs of TGroup 3 in black (Heavy):

1. Fig. 4.a) only creates one TJob from each TGroup. In this schedule, the TJobs are executed in parallel over three instances, but the test cases of the same TJobs are executed sequentially. This schedule provides a baseline, giving the worst execution

This is a pre-print of an article published in Software Quality Journal. The final authenticated version is available online at: https://doi.org/10.1007/s11219-020-09505-2 
time, but using the minimal number of instances required to keep the TGroups isolated. All TGroups are deployed in separate instances sharing the same set-up between them.

2. Fig. 4.b) also creates one TJob from each TGroup. However, the test cases of the TJob 1 are deployed in parallel over the same instance because they use the Light OpenVidu resource that allows the parallel execution of the test cases at the same time. As consequence of the parallel execution of the test cases of TJob 1, the schedule reduces the execution time in comparison with the previous one that executes them sequentially (Fig. 4.a). Each test case of the TJob 1 employs individually more execution time than by executing them sequentially due to the overload caused by the concurrent access. Nonetheless, in this case, it is not relevant because the critical execution time corresponds with the TJob 2 execution (TGroup 2).

3. Fig.4.c) as the opposite of TGroup 1, the execution time cannot be reduced executing the test cases of the TGroup 2 in parallel inside of the same instance because they cannot access concurrently to the same instance of the Medium OpenVidu resource. However, the test cases can use this resource in parallel if it is deployed in several instances. Therefore the schedule of the Fig.4.c) creates three TJobs from the TGroup 2 that are deployed in a parallel way in three instances. This schedule reduces the execution time, and the critical execution time corresponds with the TJob 3 execution (TGroup 3). However, the test cases of the TGroup 3 can not be executed in parallel at the same time neither in the same instance nor in several instances because they use the Heavy OpenVidu resource that has high elasticity cost. This schedule reduces the execution time but increases the use of resources because employs five instances.

4. Fig. 4.d) instead to create three TJobs from TGroup 2, it creates only two TJobs to reduce the number of instances. This schedule maintains the same execution time than the schedule of Fig.4.c), but also reduces the resources employed avoiding the deployment of one more instance: the schedule of Fig 4.c) deploys five instances (three Medium OpenVidu resources), whereas the schedule of Fig.4.d) only four instances (two Medium OpenVidu resources).

As shown in this working example, there are several features and constraints considered during the optimization of the test scheduling based on resource usage through test orchestration. The critical step is the proper identification of which resources are needed by the test cases and their dependencies.

\section{$5 \quad$ Evaluation}

In order to assess whether RETORCH improves the execution time and saves resources during E2E testing, we perform an empirical evaluation of the application described before. To evaluate how the resources and time may be optimized via a better distribution, we attempt to answer the following research questions:

RQ1: Does RETORCH yield an efficient execution of the E2E tests in terms of time?

This is a pre-print of an article published in Software Quality Journal. The final authenticated version is available online at: https://doi.org/10.1007/s11219-020-09505-2 
RQ2: How do the schedules proposed by RETORCH affect to the use of other resources?

Test Suite: we tested the FullTeaching application with a test suite composed of 20 tests cases available in different git repositories (Elastest Developers Team, 2017, 2018) of the Elastest/Fullteaching community. These test cases employ JUnit and Selenium Web driver to emulate the user interactions checking the main functionalities of the application: classrooms, file uploading, comment creation, among others.

Set-Up: In order to evaluate RETORCH, the test cases are executed using up to 5 HyperV virtualized instances of Ubuntu Server 18 LTS into a Ryzen 8-core, 32 gigabytes of RAM and solid-state drive computer. To analyze the efficiency of the test schedules provided by RETORCH, we measure the execution time and different memory indicators from the System Monitor: physical memory required by each instance and total physical memory required.

Resource Identification: In the FullTeaching system, we identify three different resources as indicated in Section 4: OpenVidu videoconference server (University, 2017), the Kurento media server (Technologies, 2014), and the MySQL DBMS. According to the Resource Identification detailed in Section 4, the OpenVidu resource can be replaced by another three resources depending on how the test cases use the resource: Light OpenVidu resource (mock), Medium OpenVidu resource (implementation with basic functionalities) and Heavy OpenVidu resource (full OpenVidu functionalities). When it is possible, it is preferable to execute the test cases in the Light OpenVidu resource to save resources in comparison with both Medium and Heavy OpenVidu resources, and also because the last two resources have more elasticity cost. All the resources are allocated into a Cloud server by means of a docker orchestrator (dockercompose) that also deploys the aggrupation of test cases according to the schedules proposed by RETORCH.

Grouping: The test cases are grouped into TGroups based on the resources identified. As we detailed in Section 4, we create three TGroups based on the functionality of the test cases and their usage of the OpenVidu videoconference system. The TGroup 1 is composed of the test cases that only need a session-id from the OpenVidu, then these test cases can use the Light OpenVidu resource (mock). The TGroup 2 is composed by the test cases that need OpenVidu functionality without storage, then they can use the Medium OpenVidu resource. Finally, the TGroup 3 is composed of the test cases that require the full functionality of the OpenVidu including the storage, then they can use the Heavy OpenVidu resource.

All of the test cases of the TGroup 1 use the Light OpenVidu resource with read-only access because they only request a session-id, so they should be executed sequentially/parallelized either in one instance or in several instances. In contrast, the test cases of the TGroup 2 uses the Medium OpenVidu resource with read-write access because they modify information that other test cases can access if they are executed in the same instance. To avoid issues between test cases due the concurrent access, the test cases of the TGroup 2 should be executed either sequentially in the same instance or parallelized through different instances of the Medium OpenVidu resources. However, the test cases

This is a pre-print of an article published in Software Quality Journal. The final authenticated version is available online at: https://doi.org/10.1007/s11219-020-09505-2 


\begin{tabular}{|l|l|l|l|l|}
\hline $\begin{array}{l}N^{0} \\
\text { TGroup }\end{array}$ & Resource Name & $\begin{array}{l}\text { N\# test } \\
\text { cases }\end{array}$ & $\begin{array}{l}\text { Access } \\
\text { Mode }\end{array}$ & Execution \\
\hline $\mathbf{1}$ & Light OpenVidu & 5 & Read-Only & $\begin{array}{l}\text { - Sequentially in one instance } \\
\text { - Parallel in one instance }\end{array}$ \\
\hline $\mathbf{2}$ & Medium OpenVidu & 11 & Read-Write & $\begin{array}{l}\text { - Sequentially in one instance } \\
\text { - Parallel in three instances } \\
\text { - Parallel in two instances }\end{array}$ \\
\hline $\mathbf{3}$ & Heavy OpenVidu & 4 & Read-Write & \begin{tabular}{l} 
- Sequentially in one instance \\
\hline
\end{tabular} \\
& & & & \\
\hline
\end{tabular}

Table 1 FullTeaching test cases

of the TGroup 2 should not be executed in parallel way in the same instance of the Medium OpenVidu resource. Finally, all the test cases of TGroup 3 also use OpenVidu with read-write access, but in contrast, they use the Heavy OpenVidu resource because they need storage to modify and access to the data. To avoid issues between the test cases due the concurrent access, the test cases of the TGroup 3 should be executed sequentially in the same instance. However, the test cases of the TGroup 3 should not be executed in several instances of the Heavy OpenVidu resources because the resource has a high elastic cost.

Table 1 summarizes the number of test cases per each TGroup and their possible executions. The TGroup 1 has 5 test cases, TGroup 2 has 11 test cases, and the TGroup 3 has 4 test cases. The test cases of these TGroups will be executed in TJobs according to the scheduling.

Scheduling: We have executed the four schedules that are detailed in Section 4 and represented in Fig. 4. The first schedule A creates one TJob per each TGroup, that means that the TJob 1 is created with all test cases of TGroup 1 (5 test cases), the TJob 2 with all of TGroup 2 (11 test cases), and the TJob 3 with all of TGroup 3 (4 test cases). The three TJobs of schedule A are executed in parallel each one in one instance. Despite the TJobs are executed in parallel, the schedule A proposes that the test cases of each TJob should be executed sequentially in the instance of the TJob. In contrast, schedule B proposes that the test cases of the TGroup 1 should be executed in the same TJob using one instance but the test cases in parallel. The schedule $\mathrm{C}$ proposes to parallelize the execution of the TGroup 2 in 3 TJobs executing each one in one instance. Finally, the schedule D instead to parallelize the execution of the test cases of TGroup 2 in three instances, parallelizes them in two instances. Once these four schedules are executed, we answer the research questions analyzing the efficiency of the test execution: memory required by the virtualized instances and execution time of the test cases.

This is a pre-print of an article published in Software Quality Journal. The final authenticated version is available online at: https://doi.org/10.1007/s11219-020-09505-2 


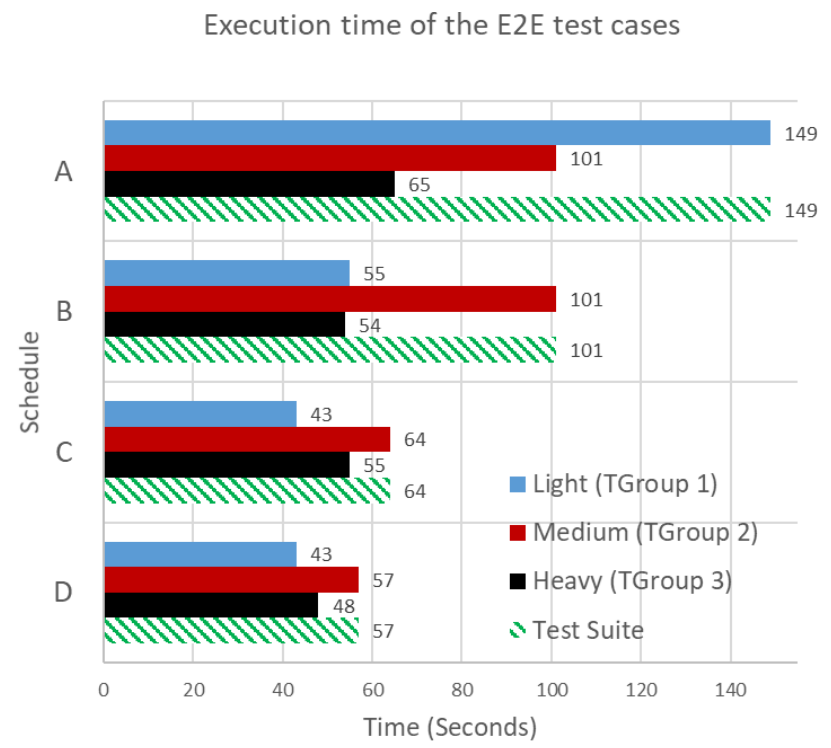

Fig. 5. Execution time different schedules

RQ1: To evaluate how RETORCH may optimize the execution time of the test suite, we have executed the schedules and obtained the execution time on the up to five virtualized instances. Fig. 5. depicts the execution time of the E2E tests cases with the four schedules represented in Fig.4. (A, B, C and D). The execution time is obtained from the test log timestamps. The blue lines represent the execution time of the TGroup 1, the red about TGroup 2, the black about the TGroup 3, and the green/white the total execution time of the test suite according to the schedule.

RETORCH reduces the execution time of the test suite by a $61.74 \%$ (from 149 seconds in the schedule A to 57 seconds in the schedule D). The schedule A employs 149 seconds because the test cases of TGroup 1 are executed sequentially during these 149 seconds. The schedule B execute these test cases in parallel inside of the same instance reducing the execution time of the test cases of TGroup 1 to 55 seconds. However, the execution time of schedule $B$ is 101 seconds because the sequential execution of the test cases of TGroup 2 takes 101 seconds. The schedule $C$ executes the test cases of the TGroup 2 in parallel in three instances reducing the execution time to 64 seconds. Finally, Schedule D executes the test cases of the TGroup 2 in two instances to optimize the resource usage employing 57 seconds. According to the FullTeaching system and the four schedules evaluated, RETORCH is able to reduce the execution time more than half through the identification of the resources, grouping of the test cases and the sequential/parallel scheduling according to test dependencies.

RQ2: To evaluate the performance in terms of resource usage, we monitor the physical memory used in all the virtualized instances during the testing. This measurement was obtained via the Hyper-V performance monitor, that provides the memory requested by each instance together with the percentage of memory used at each moment.

This is a pre-print of an article published in Software Quality Journal. The final authenticated version is available online at: https://doi.org/10.1007/s11219-020-09505-2 
With these two values, we obtain the memory used by the different virtualized instances at every moment multiplying the percentage of use by the memory requested. The resource usage in the four schedules is represented in Fig. 6. The X-axis represents the execution time and $\mathrm{Y}$-axis the total amount of the memory used by all the instances in gigabytes. Note that the four schedules represented in the figure employ different execution times. Schedule A takes 149 seconds, but the schedule D finishes at 57 seconds. During these seconds the memory usage varies in different ways, sometimes with peaks and other times flatter depending on the sequential/parallel execution of the test cases and instances. The schedule A executes the test cases in 3 instances, the schedule B also in 3 instances, the schedule $\mathrm{C}$ in 5 instances, and the schedule $\mathrm{D}$ in 4 instances.

RETORCH reduces the execution time executing the test cases in parallel either in one instance or several instances. The increasing of one instance also increases the memory usage, but RETORCH provides a schedule that at the same time that reduces the execution time also optimizes the usage of memory. We can observe from the schedule A to schedule D that RETORCH reduces the execution time by $61.74 \%$ (RQ1) whereas the memory usage only increases a $\sim \mathrm{x} 2$ (from $\sim 8 \mathrm{~GB}$ to $\sim 15 \mathrm{~GB}$ in the lower values, and from $\sim 12 \mathrm{~GB}$ to $\sim 26 \mathrm{~GB}$ in the peak). Despite the memory increases, RETORCH optimizes the memory usage at the same time that aims to reduce the execution time.

The schedule A executes in parallel three TJobs in three instances employing $\sim 15 \mathrm{~GB}$. All test cases of each TJobs are executed sequentially according to the Schedule $\mathrm{A}$, so the memory usage is more or less flat (the lower value is $\sim 8 \mathrm{~GB}$, but usually is around $\sim 15 \mathrm{~GB}$ ). Schedule B instead to execute the test cases of TGroup 1 sequentially in one instance, proposes to execute them in parallel in the one instance. The parallel execution of these test cases not only reduces the execution time but also increases the memory achieving a peak of $\sim 24 \mathrm{~GB}$ when the test cases of TGroup 1 are executed in parallel at the same time. Schedule $C$ proposes to execute the test cases of TGroup 2 in parallel in three instances. The parallel execution of the test cases again

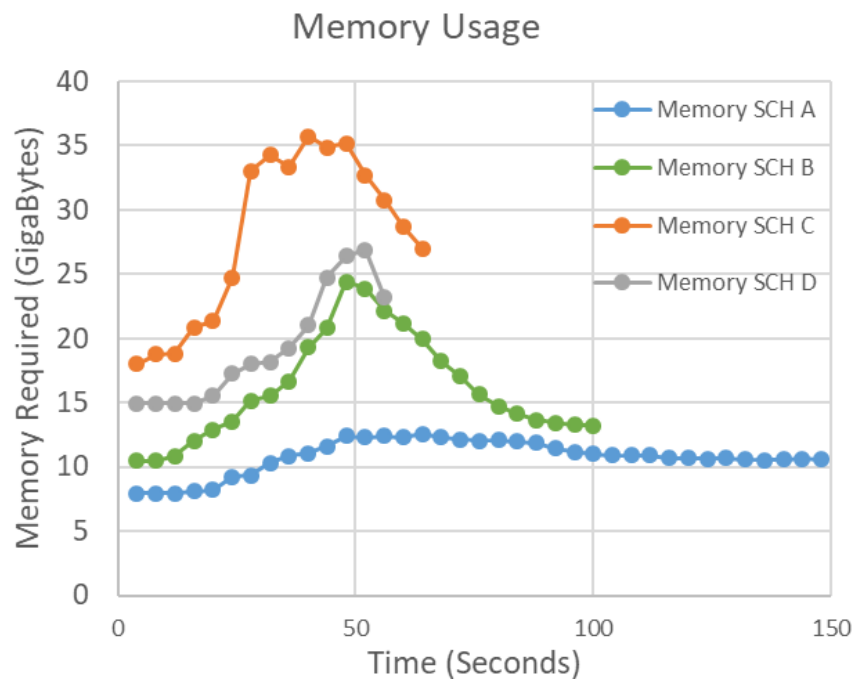

$\mathrm{T}$

Fig. 6. Memory Usage in the different Schedules

version is available online at: https://doi.org/10.1007/s11219-020-09505-2 
decreases the execution time but also increases the memory achieving some peaks of $\sim 36 \mathrm{~GB}$. The memory has increased a lot because each instance of the three deployed for the test cases of TGroup 2 contains the Medium Openvidu resource, Kurento media server resource, and MySQL database resource. To optimize the resources, the schedule D proposes to reduce one instance for the test cases of TGroup 2, that is to execute two instances for the test cases of TGroup 2, one instance for TGroup 1 and another instance for TGroup 3. The reduction of one instance from schedule $\mathrm{C}$ to $\mathrm{D}$ not only improves the execution time but also reduces/optimizes the memory usage from $\sim 18 \mathrm{~GB}$ to $\sim 15 \mathrm{~GB}$ in lower values and from $\sim 36 \mathrm{~GB}$ to $\sim 26 \mathrm{~GB}$ during the peak. According to the FullTeaching system and the four schedules evaluated, RETORCH not only reduces the execution time of E2E testing through the sequential/parallel execution but also optimizes the resource usage varying the number of instances considering the resources deployed.

Threats to validity: The above evaluation shows promising results of the RETORCH approach. However, there are several issues that may threaten the validity of these results. Regarding the internal threats, the evaluation analyzes the memory usage and execution time on which it is easy to introduce noise into the measures by other system processes. In order to mitigate this issue, we performed the experimentation into the same dedicated computer inside virtualized instances with the same specifications. Regarding the external threats, that may limit the ability to generalize the results, our evaluation is related to only one case of study with a limited set of resources. Despite this, the results provide us insights that by carefully arranging the resources used by the test cases improve the overall efficiency of the test execution may be improved, although more experimentation should be done with other systems and different kinds of resources. Another issue is related to the size of the test suite. Although the test suite is not large, it contains a variety of typical tests scenarios in E2E testing, which have been taken from a real-world application. Finally, regarding the construct validity, we handled the most representative variables (e.g. overall test execution time and memory consumption). Other measures have not been considered (e.g. processor load). To mitigate this problem, we have monitored the other resources, observing that the other resources remain with a low usage rate compared with the memory.

\section{Conclusions and future work}

This article proposes an approach called RETORCH to orchestrate the execution of the End-to-End test cases (E2E) through: the identification of resources required to run an E2E test case, the grouping of the test cases based on the minimization of the resources to be deployed and on the parallel scheduling of the tests in several instances. We performed an evaluation of RETORCH with a real-world application in a Cloud test environment.

The results show that RETORCH improves the efficiency of the E2E test execution optimizing their resources and execution time. The execution time is decreased through the scheduling of the test cases in several instances considering the test dependencies

This is a pre-print of an article published in Software Quality Journal. The final authenticated version is available online at: https://doi.org/10.1007/s11219-020-09505-2 
and other issues related to parallelize the test execution. Not only the execution of the tests in several instances increases the usage of resources like memory, but RETORCH also optimizes these resources avoiding the oversubscription that may cause re-deployments through the aggrupation of similar tests in the same instance.

As future work, we plan to integrate RETORCH in the ElasTest platform to orchestrate efficiently the execution of the E2E test cases. This would require the automatic identification of both resources and dependencies between the tests in the cloud systems. Another line of research pursues to thoroughly evaluate the grouping and scheduling methods in the context of optimizing the E2E test executions.

\section{References}

Augusto, C., Morán, J., Bertolino, A., de la Riva, C., \& Tuya, J. (2019). RETORCH: Resourceaware End-to-end Test Orchestration. In M. Piattini, P. Rupino da Cunha, I. GarcíaRodríguez de Guzmán, \& R. Pérez-Castillo (Eds.), 12th International Conference on the Quality of Information and Communications Technology (QUATIC 2019) (p. 14). https://doi.org/10.1007/978-3-030-29238-6_22

Bell, J., Kaiser, G., Melski, E., \& Dattatreya, M. (2015). Efficient dependency detection for safe Java test acceleration. 2015 10th Joint Meeting of the European Software Engineering Conference and the ACM SIGSOFT Symposium on the Foundations of Software Engineering, ESEC/FSE $2015 \quad$ - $\quad$ Proceedings, 770-781. https://doi.org/10.1145/2786805.2786823

Bertolino, A. (2007). Software testing research: Achievements, challenges, dreams. FoSE 2007: Future of Software Engineering, 85-103. https://doi.org/10.1109/FOSE.2007.25

Bertolino, A., Calabró, A., De Angelis, G., Gallego, M., García, B., \& Gortázar, F. (2018). When the testing gets tough, the tough get ElasTest. Proceedings - International Conference on Software Engineering, 17-20. https://doi.org/10.1145/3183440.3183497

Bertolino, A., de Angelis, G., Gallego, M., García, B., Gortázar, F., Lonetti, F., \& Marchetti, E. (2019). A Systematic Review on Cloud Testing. ACM Computing Surveys, 52(5), 1-42. https://doi.org/10.1145/3331447

Burns, B., Grant, B., Oppenheimer, D., Brewer, E., \& Wilkes, J. (2016). Borg, omega, and kubernetes. Communications of the ACM, 59(5), 50-57. https://doi.org/10.1145/2890784

Casalicchio, E. (2017). Autonomic orchestration of containers: Problem definition and research challenges. ValueTools 2016 - 10th EAI International Conference on Performance Evaluation Methodologies and Tools, 287-290. https://doi.org/10.4108/eai.25-102016.2266649

Chakraborty, S. S., \& Shah, V. (2011). Towards an approach and framework for test-execution plan derivation. 2011 26th IEEE/ACM International Conference on Automated Software Engineering, ASE 2011, Proceedings, 488-491. https://doi.org/10.1109/ASE.2011.6100106

De Brito, M. S., Hoque, S., Magedanz, T., Steinke, R., Willner, A., Nehls, D., ... Schreiner, F. (2017). A service orchestration architecture for Fog-enabled infrastructures. 2017 2nd International Conference on Fog and Mobile Edge Computing, FMEC 2017, 127-132. https://doi.org/10.1109/FMEC.2017.7946419

Docker Inc. (2017). Overview of Docker Compose | Docker Documentation. Retrieved October

This is a pre-print of an article published in Software Quality Journal. The final authenticated version is available online at: https://doi.org/10.1007/s11219-020-09505-2 
14, 2019, from Docker Inc. website: https://docs.docker.com/compose/

Docker Inc. (2019). Swarm mode overview | Docker Documentation. Retrieved October 15, 2019, from https://docs.docker.com/engine/swarm/

Draft, W. (2014). TOSCA Simple Profile in YAML Version 1.0. (March), 1-83. Retrieved from http://docs.oasis-open.org/tosca/TOSCA-Simple-Profile-YAML/v1.1/TOSCA-SimpleProfile-YAML-v1.1.html

Elastest Developers Team. (2017). Elastest: Full-teaching. Retrieved October 28, 2019, from https://github.com/elastest/full-teaching

Elastest Developers Team. (2018). Elastest: FullTeaching-experiment. Retrieved October 28, 2019, from https://github.com/elastest/full-teaching-experiment

Engström, E., Skoglund, M., \& Runeson, P. (2008). Empirical evaluations of regression test selection techniques: A systematic review. ESEM'08: Proceedings of the 2008 ACM-IEEE International Symposium on Empirical Software Engineering and Measurement, 22-31. https://doi.org/10.1145/1414004.1414011

Esfahani, H., Fietz, J., Ke, Q., Kolomiets, A., Lan, E., Mavrinac, E., ... Kandula, S. (2016). CloudBuild: Microsoft's distributed and caching build service. Proceedings International Conference on Software Engineering, 11-20. https://doi.org/10.1145/2889160.2889222

Fitzgerald, B., \& Stol, K. J. (2017). Continuous software engineering: A roadmap and agenda. Journal of Systems and Software, 123, 176-189. https://doi.org/10.1016/j.jss.2015.06.063

Gambi, A., Bell, J., \& Zeller, A. (2018). Practical Test Dependency Detection. Proceedings 2018 IEEE 11th International Conference on Software Testing, Verification and Validation, ICST 2018, 1-11. https://doi.org/10.1109/ICST.2018.00011

Gambi, A., Gorla, A., \& Zeller, A. (2017). O!Snap: Cost-Efficient Testing in the Cloud. Proceedings - 10th IEEE International Conference on Software Testing, Verification and Validation, ICST 2017, 454-459. https://doi.org/10.1109/ICST.2017.51

Garcia, B., Lonetti, F., Gallego, M., Miranda, B., Jimenez, E., De Angelis, G., .. Marchetti, E. (2018). A proposal to orchestrate test cases. Proceedings - 2018 International Conference on the Quality of Information and Communications Technology, QUATIC 2018, 38-46. https://doi.org/10.1109/QUATIC.2018.00016

Giotis, K., Kryftis, Y., \& Maglaris, V. (2015). Policy-based orchestration of NFV services in Software-Defined Networks. 1st IEEE Conference on Network Softwarization: SoftwareDefined Infrastructures for Networks, Clouds, IoT and Services, NETSOFT 2015, 1-5. https://doi.org/10.1109/NETSOFT.2015.7116145

Gyori, A., Shi, A., Hariri, F., \& Marinov, D. (2015). Reliable testing: Detecting state-polluting tests to prevent test dependency. 2015 International Symposium on Software Testing and Analysis, ISSTA 2015 - Proceedings, 223-233. https://doi.org/10.1145/2771783.2771793

Harman, M. (2011). Making the case for MORTO: Multi objective regression test optimization. Proceedings - 4th IEEE International Conference on Software Testing, Verification, and Validation Workshops, ICSTW 2011, 111-114. https://doi.org/10.1109/ICSTW.2011.60

Herzig, K., Greiler, M., Czerwonka, J., \& Murphy, B. (2015). The art of testing less without sacrificing quality. Proceedings - International Conference on Software Engineering, 1, 483-493. https://doi.org/10.1109/ICSE.2015.66

Lachmann, R., Nieke, M., Seidl, C., Schaefer, I., \& Schulze, S. (2017). System-level test case prioritization using machine learning. Proceedings - 2016 15th IEEE International

This is a pre-print of an article published in Software Quality Journal. The final authenticated version is available online at: https://doi.org/10.1007/s11219-020-09505-2 
Conference on Machine Learning and Applications, ICMLA 2016, 361-368. https://doi.org/10.1109/ICMLA.2016.163

Liu, C. H., Chen, S. L., \& Chen, W. K. (2017). Cost-benefit evaluation on parallel execution for improving test efficiency over cloud. Proceedings of the 2017 IEEE International Conference on Applied System Innovation: Applied System Innovation for Modern Technology, ICASI 2017, 199-202. https://doi.org/10.1109/ICASI.2017.7988384

Memon, A., Gao, Z., Nguyen, B., Dhanda, S., Nickell, E., Siemborski, R., \& Micco, J. (2017). Taming google-scale continuous testing. Proceedings - 2017 IEEE/ACM 39th International Conference on Software Engineering: Software Engineering in Practice Track, ICSE-SEIP 2017, 233-242. https://doi.org/10.1109/ICSE-SEIP.2017.16

Meyer, M. (2014). Continuous integration and its tools. IEEE Software, 31(3), 14-16. https://doi.org/10.1109/MS.2014.58

Microsoft. (n.d.). Orchestrator overview | Microsoft Docs. Retrieved October 15, 2019, from https://docs.microsoft.com/en-us/system-center/orchestrator/learn-aboutorchestrator?view=sc-orch-2019

Oracle. (2019). MySQL. Retrieved November 3, 2019, from https://www.mysql.com/

Pérez, P. F. (2017). Fullteaching: A web application to make teaching online easy. Retrieved from https://github.com/pabloFuente/full-teaching

Rothermel, G., Harrold, M. J., Von Ronne, J., \& Hong, C. (2002). Empirical studies of test-suite reduction. Software Testing Verification and Reliability, 12(4), 219-249. https://doi.org/10.1002/stvr.256

Shull, F., Basili, V., Boehm, B., Brown, A. W., Costa, P., Lindvall, M., ... Vinter, O. (2002). What we have learned about fighting defects. Proceedings - International Software Metrics Symposium, 2002-Janua, 249-258. https://doi.org/10.1109/METRIC.2002.1011343

Singh, S., \& Chana, I. (2015). QoS-aware autonomic resource management in cloud computing: A systematic review. ACM Computing Surveys, 48(3), 1-46. https://doi.org/10.1145/2843889

Technologies, K. (2014). Kurento. Retrieved from https://www.kurento.org/

Toczé, K., \& Nadjm-Tehrani, S. (2018, June 4). A Taxonomy for Management and Optimization of Multiple Resources in Edge Computing. Wireless Communications and Mobile Computing, Vol. 2018, pp. 1-23. https://doi.org/10.1155/2018/7476201

Uberti, J., \& Thatcher, P. (2018). \{WebRTC\} Home Nextbar\} \{WebRTC\}. Retrieved from https://webrtc.org/

University, R. J. C. (2017). OpenVidu. Retrieved from https://openvidu.io/

Velasquez, K., Abreu, D. P., Assis, M. R. M., Senna, C., Aranha, D. F., Bittencourt, L. F., ... Madeira, E. (2018). Fog orchestration for the Internet of Everything: state-of-the-art and research challenges. Journal of Internet Services and Applications, 9(1), 14. https://doi.org/10.1186/s13174-018-0086-3

Velasquez, K., Abreu, D. P., Goncalves, D., Bittencourt, L., Curado, M., Monteiro, E., \& Madeira, E. (2017). Service orchestration in fog environments. Proceedings - 2017 IEEE 5th International Conference on Future Internet of Things and Cloud, FiCloud 2017, 2017-Janua, 329-336. https://doi.org/10.1109/FiCloud.2017.49

Wong, W. E., Morgan, J. R., London, S., \& Mathur, A. P. (1998). Effect of test set minimization on fault detection effectiveness. Software - Practice and Experience, 28(4), 347-369.

This is a pre-print of an article published in Software Quality Journal. The final authenticated version is available online at: https://doi.org/10.1007/s11219-020-09505-2 
https://doi.org/10.1002/(SICI)1097-024X(19980410)28:4<347::AID-SPE145>3.0.CO;2$\mathrm{L}$

Yoo, S., \& Harman, M. (2012, March). Regression testing minimization, selection and prioritization: A survey. Software Testing Verification and Reliability, Vol. 22, pp. 67120. https://doi.org/10.1002/stv.430

Yu, L., Su, Y., \& Wang, Q. (2009). Scheduling test execution of WBEM applications. Proceedings - Asia-Pacific Software Engineering Conference, APSEC, 323-330. https://doi.org/10.1109/APSEC.2009.27

Zhang, Z., Li, C., Tao, Y., Yangy, R., Tang, H., \& Xu, J. (2014). Fuxi: A fault-tolerant resource management and job scheduling system at internet scale. Proceedings of the VLDB Endowment, 7(13), 1393-1404. https://doi.org/10.14778/2733004.2733012

This is a pre-print of an article published in Software Quality Journal. The final authenticated version is available online at: https://doi.org/10.1007/s11219-020-09505-2 